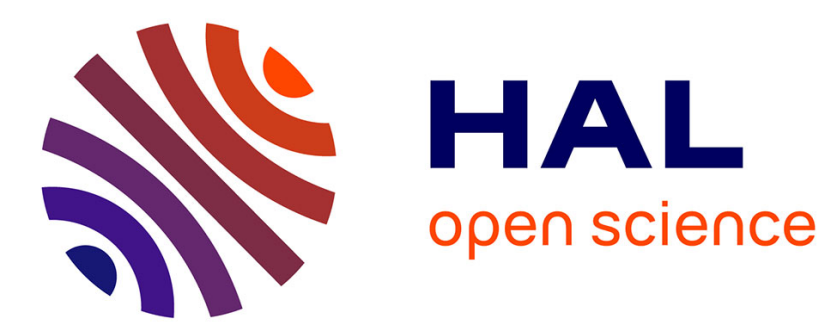

\title{
La route des Flandres. L'exil bruxellois du duc d'York et la crise de l'Exclusion (1679)
}

Charles-Edouard Levillain

\section{To cite this version:}

Charles-Edouard Levillain. La route des Flandres. L'exil bruxellois du duc d'York et la crise de l'Exclusion (1679). Dix-septième siècle, 2012, 257, pp.663-679. 10.3917/dss.124.0663 . hal-03251110

\author{
HAL Id: hal-03251110 \\ https://hal.science/hal-03251110
}

Submitted on 10 Jun 2021

HAL is a multi-disciplinary open access archive for the deposit and dissemination of scientific research documents, whether they are published or not. The documents may come from teaching and research institutions in France or abroad, or from public or private research centers.
L'archive ouverte pluridisciplinaire HAL, est destinée au dépôt et à la diffusion de documents scientifiques de niveau recherche, publiés ou non, émanant des établissements d'enseignement et de recherche français ou étrangers, des laboratoires publics ou privés. 


\title{
LA ROUTE DES FLANDRES. L'EXIL BRUXELLOIS DU DUC D'YORK ET LA CRISE DE L'EXCLUSION (1679)
}

\author{
Charles-Édouard Levillain
}

P.U.F. | Dix-septième siècle

$2012 / 4-n^{\circ} 257$

pages 663 à 679

ISSN 0012-4273

Article disponible en ligne à l'adresse:

http://www.cairn.info/revue-dix-septieme-siecle-2012-4-page-663.htm

Pour citer cet article :

Levillain Charles-Édouard, « La route des Flandres. L'exil bruxellois du duc d'York et la crise de l'Exclusion (1679) », Dix-septième siècle, 2012/4 n²57, p. 663-679. DOI : 10.3917/dss.124.0663

Distribution électronique Cairn.info pour P.U.F..

(c) P.U.F.. Tous droits réservés pour tous pays.

La reproduction ou représentation de cet article, notamment par photocopie, n'est autorisée que dans les limites des conditions générales d'utilisation du site ou, le cas échéant, des conditions générales de la licence souscrite par votre établissement. Toute autre reproduction ou représentation, en tout ou partie, sous quelque forme et de quelque manière que ce soit, est interdite sauf accord préalable et écrit de l'éditeur, en dehors des cas prévus par la législation en vigueur en France. II est précisé que son stockage dans une base de données est également interdit. 


\section{La route des Flandres. L'exil bruxellois du duc d'York et la crise de l'Exclusion (1679)*}

Après trois années marquées par un regain d'intérêt pour la Glorieuse Révolution (1688), il est sans doute temps pour les historiens du second $\mathrm{XvII}^{\mathrm{e}}$ siècle de revenir à la crise de l'Exclusion $(1678-1681)^{1}$ : une longue crise politicoreligieuse de trois ans qui, coincée entre deux révolutions, a échappé au vigoureux débat entre George Trevelyan et Christopher Hill à la fin des années 1930 avant de se chercher une identité et un sens dans l'historiographie plus récente, quelque part entre réitération de la guerre civile et annonce de la Glorieuse

* Je tiens à remercier Stéphane Jettot de son invitation à publier cet article dans le présent volume. Mes remerciements vont également à la Casa de Velázquez (et notamment à Jean-Pierre Étienvre et Stéphane Michonneau) qui, en m’accueillant comme boursier au printemps 2011, m’ont permis de faire les recherches nécessaires à cette étude. L'Angleterre utilisa jusqu'en 1751 un calendrier julien, dit " ancien style " (Old Style), en retard de dix jours sur le calendrier grégorien. De plus, l'année commençait le 25 mars. Nous avons choisi d'adopter le calendrier ancien style, mais en faisant commencer l'année le $1^{\text {er }}$ janvier. Lorsqu'il sera question d'un courrier envoyé d'un pays autre que l'Angleterre, nous donnerons la date du calendrier "nouveau style " (New Style) en ajoutant la mention NS. Pour éviter toute confusion possible entre Charles II d'Angleterre et Charles II d'Espagne, nous écrirons " Charles II " pour le premier et " Carlos II " pour le second. Abréviations utilisées : AD : Archives départementales. AGDHC : Anchitell Grey, Debates of the House of Commons from the Year 1667 to the Year 1694, Londres, 1763, 9 volumes. AGR : Bruxelles : Archives générales du royaume. AGS : Simancas, Archivo General de Stato. BL : Londres, The British Library. BNM : Madrid, Biblioteca Nacional. CUL : Cambridge University Library. EHR : The English Historical Review. HDNP : Amsterdam, Historisch Documentatiecentrum voor het Nederlandse Protestantisme. HJ : The Historical Journal. KB : La Haye, Koninklijke Bibliotheek. NA : La Haye, Nationaal Archief. TBL: New Haven, The Beinecke Library. TNA: Kew, The National Archives.

1. Steven Pincus, 1688. The First Modern Revolution, New Haven, Yale University Press, 2009. Gabriel Glickman, The English Catholic community, 1688-1745: Politics, Culture and Ideology, Woodbridge, Boydell \& Brewer, 2009. Charles-Édouard Levillain, "Thomas Macaulay ou comment s'en débarrasser. Nouvelles perspectives historiographiques sur la Glorieuse Révolution (1688) : autour d'un ouvrage de Steven Pincus ", Histoire, Économie et Société, n 1, 2011, pp. 1-20. 
Révolution ${ }^{2}$. Contrairement à l'historiographie de la Glorieuse Révolution, celle de la crise de l'Exclusion est restée obstinément insulaire, faisait très peu de cas des événements du continent et ignorant superbement les archives non-britanniques à l'exception, évidemment, de la correspondance Barillon, que les historiens pratiquent depuis longtemps ${ }^{3}$. Un seul exemple: Jonathan Scott, le moins insulaire des spécialistes de la crise de l'Exclusion, consacre finalement très peu de place au contexte européen des années 1678-1681, pourtant marquées par des événements aussi essentiels que la fin de la guerre de Hollande (1672-1678), les longues négociations des traités de Nimègue (1675-1679) et le début de la guerre des "réunions " ${ }^{4}$. Péché d'insularité ? Le reproche serait facile. La crise de l'Exclusion reste si singulière dans ses origines et son déroulement qu'il n'est pas interdit d'y voir le symptôme d'une exception britannique, ne serait-ce que dans cet écartèlement maximal entre imaginaire et réalité du complot ${ }^{5}$ : rien, en dehors d'une dimension dynastique certaine, qui puisse a priori être rattaché à la fameuse « crise générale du XVII ${ }^{\mathrm{e}}$ siècle " décrite par Hugh Trevor-Roper dans les années $1950^{6}$.

La présente étude se fixe principalement deux objectifs : d'abord, ouvrir l'historiographie de la crise de l'Exclusion à l'histoire plus large du continent et notamment des Flandres espagnoles qui, malgré leur proximité géographique avec les côtes anglaises de l'Angleterre, ont été occultées par les études existantes ; ensuite, à l'intérieur de ce cadre anglo-espagnol, examiner une période oubliée de la vie du duc d'York, frère cadet de Charles II et futur Jacques II : son exil bruxellois au cours du printemps et de l'été $1679^{7}$. À travers la situation particulière du duc d'York, se pose la question plus large d'un étonnant jeu à quatre puissances qui explique en partie la sinuosité des négociations de Nimègue : d'un côté un tandem hispano-néerlandais mis en place en $1673^{8}$, de l'autre une puissance française qui, depuis 1667 ,

2. Jonathan Scott, England's Troubles. Seventeenth-Century Political Instability in European Context, Cambridge, Cambridge University Press, 2000. John Miller, After the Civil Wars. English Politics and Government in the Reign of Charles II, Londres, Longman, 2000. Tim Harris, Charles II and his kingdoms 1660-1685, Londres, Allen Lane, 2005. Sur Shaftesbury en particulier, le leader des Whigs, cf. Anthony Ashley Cooper First Earl of Shaftesbury, 1621-1683, éd. John Spurr, Aldershot, Ashgate, 2011. Pour le débat Trevelyan/Hill, cf. Christopher Hill, The English Revolution, Londres, Lawrence \& Wishart, 1940. Christopher Hill réagissait à une étude de George Trevelyan publiée en 1938 qui considérait que l'Angleterre avait connu sa vraie révolution en 1688-1689 et non en 1640-1642. G.M. Trevelyan, The English Revolution, 1688-1689, Londres, Thornton Butterworth, 1938.

3. Sir John Dalrymple, Memoirs of Great-Britain and Ireland, from the Dissolution of the last Parliament of Charles II until the sea-battle of La Hogue, Londres, 1771.

4. Pour les négociations de Nimègue, cf. La paix de Nimègue 1676-1678/1679. Colloque international du Tricentenaire, éd. Hans Bots, Amsterdam, APA-Holland University Press, 1980. Pour le contexte des guerres de "réunion ", cf. Bertrand Jeanmougin, Louis XIV à la conquête des Pays-Bas espagnols. La guerre oubliée 1678-1684, Paris, Economica, 2005.

5. René Pillorget, "Le complot papiste dans l'imaginaire anglais au dix-septième siècle ", Storia della storiografia, volume XIV, 1988, pp. 119-135.

6. Hugh R. Trevor-Roper, "The general crisis of the seventeenth-century ", Past \& Present, n 16, 1959, pp. 31-64.

7. Il n'en est pour ainsi dire pas question dans les biographies récentes de Jacques II. Cf. John Miller, James II, New Haven et Londres, Yale University Press, 2000. W.A. Speck, James II, Londres, Longman, 2002.

8. Manuel Herrero Sánchez, El acercamiento hispano-neerlandés 1648-1678, Madrid, 2000. 
ne cessait de mettre en péril la situation fragile des Pays-Bas espagnols; au milieu, un roi d'Angleterre tiraillé entre des forces contraires : son alliance officielle avec la Hollande (depuis 1677), son alliance officieuse avec la France (depuis 1670) et un Parlement rebelle qui, depuis 1673, lui menait la vie dure, cherchant précisément à détacher pour de bon l'Angleterre de l'influence de Louis XIV. À partir du déclenchement de la crise de l'Exclusion, une seule question se posa au roi : comment survivre? Plus précisément : comment éviter de devoir consentir à l'exclusion de son frère catholique de la succession protestante?

Face à une fronde parlementaire dont la vigueur et l'intensité ravivaient les mauvais souvenirs de 1640-1642, Charles II usa de deux contre-feux : d'un côté son contrôle souverain du calendrier parlementaire ; de l'autre son droit d'aînesse, en quelque sorte, qui lui permit d'imposer une période d'exil à son frère cadet, d'abord à Bruxelles, puis à Édimbourg. Charles II n'était ni particulièrement philosophe ni particulièrement sentimental; plutôt pragmatique, armé d'un bon sens et d'un instinct de survie hérités du traumatisme des années 1650, où il avait erré sur le continent en quête d'aumônes, entouré d'une Cour réduite à une peau de chagrin. Le problème venant du duc d'York, on le sortirait donc d'Angleterre en attendant que passe l'orage. Suivons maintenant ce prince catholique sur la route des Flandres pour comprendre ce qui se déroula à Bruxelles au printemps et à l'été 1679.

\section{ANGLeterRE-EsPAGNE : GÉOPOLITIQUE DE LA RÉCONCILIATION}

\section{Les Flandres espagnoles, "frontière naturelle de l'Angleterre " (Marvell)}

Après la percée fulgurante des armées françaises vers les Flandres au cours de la guerre de Dévolution (1667-1668), l'Espagne, l'Angleterre et la Hollande partagèrent une préoccupation sécuritaire majeure : l'intégrité territoriale des Pays-Bas espagnols, cette bande étroite qui s'étirait grosso modo du Fort de Charlemont à Anvers et qui devait servir de "barrière " entre la France et ses voisins immédiats (Espagne et Hollande) dès l'ouverture de la querelle de la succession d'Espagne $^{9}$. Successeur du marquis de Bourgomaine en qualité d'ambassadeur d'Espagne à Londres en 1679, Don Pedro Ronquillo considérait que les Pays-Bas espagnols, loin de se réduire à des provinces du Nord, impliquaient les intérêts de la monarchie espagnole tout entière ${ }^{10}$. La logique qui, sous Philippe II, avait présidé à la défense des Pays-Bas contre les rebelles néerlandais, restait la même : perdre ne serait-ce qu'une portion des Flandres espagnoles revenait à un démembrement de la monarchie. Un rapport envoyé en 1681 au Prince d'Orange n'hésitait pas, pour

9. Pour une étude de géographie historique et juridique sur la "barrière ", cf. Nelly Girard d'Albissin, Genèse de la frontière franco-belge. Les variations des limites septentrionales de la France de 1659 à 1789 , Paris, Picard, 1970. Nous renvoyons également à l'étude de référence de l'historien belge Hubert Van Houtte, Les occupations étrangères en Belgique sous l'Ancien Régime, Gand, 1930.

10. AGS, Estado 3868, Don Pedro Ronquillo à Carlos II, 25 août 1681, s.f. 
le convaincre de la nécessité d'une assistance militaire contre la France, à comparer la province des Flandres à une "Inde ${ }^{11}$ ".

Ces "Indes espagnoles en Europe " revêtaient une importance stratégique non moins grande pour l'Angleterre. Ce fut d'ailleurs là une remarquable constante dans la politique étrangère britannique, depuis le traité de Non-Such de 1585 jusqu'aux années 1930 : les Pays-Bas constituaient ce que le poète et publiciste Andrew Marvell appela en 1677 une "frontière naturelle de l'Angleterre ${ }^{12}$ ", une sorte d'avant-poste qui servait de dernière ceinture défensive avant la barrière maritime qui séparait Anvers (ou Ostende) de Douvres. Comme le résumait l'homme d'affaires arménien Calouste Gulbenkian à l'intention du Premier ministre Hendrikus Colijn en 1937 : laisser une puissance étrangère occuper le port d'Anvers eût constitué un revirement par rapport à une politique qui durait depuis le règne d'Élisabeth ${ }^{13}$. Défendre les Flandres pour défendre l'Angleterre : la problématique resta pratiquement inchangée pendant trois siècles et demi.

Dans les années 1670, la guerre de Hollande poussa l'Angleterre et l'Espagne dans les bras l'une de l'autre autour de la protection des Flandres, dont Manuel de Lira nota à juste titre qu'elles étaient « sans frontières » en raison de leurs faibles capacités défensives ${ }^{14}$. En réponse à un pamphlet anti-français, publié en 1677, qui accusait Charles II d'agir " en vrai partisan de la France " et de faire courir un danger à toute l'Europe en se laissant guider sa conduite par Louis XIV ${ }^{15}$, un dialogue imaginaire ironisa sur le rapprochement soudain entre deux puissances ennemies qui s'étaient si durement affrontées à la fin du XvI siècle : "Pensent-ils ", interrogea l'un des personnages nommé Pierre, " qu'on ne se souvient pas de la flotte nommée l'Invincible et du dessein de Philippe II en 1588 d'envahir l'Angleterre ${ }^{16}$ ? " L'ennemi, désormais, n'était plus l'Espagne mais la France. Dans une perspective anglonéerlandaise, Louis XIV ne faisait que s'inscrire dans la continuité de Philippe II, cherchant à "pacifier l'humanité" par la guerre ${ }^{17}$. Depuis ce remarquable poste

11. AGS, Estado 3868, Papier de Fernand Laberne envoyé au Prince d'Orange, rapportant ce qu'il y a à faire pour assurer le salut des Pays-Bas, 17 septembre 1681, s.f.

12. Andrew Marvell, An Account of the Growth of Popery and Arbitrary Government (1678), The Prose Works of Andrew Marvell, éd. Annabel Patterson, Nicholas von Maltzahn et N.H. Keeble, New Haven et Londres, Yale University Press, 2003, vol. II, p. 242. Pour une mise au point récente sur le contexte international qui présida à la publication de ce célèbre pamphlet, cf. Charles-Édouard Levillain, "Anti-French discourse and Country culture in Andrew Marvell ", New Perspectives on Andrew Marvell, éd. Gilles Sambras, Reims, Presses universitaires de Reims, 2008, pp.113-128. Sur Marvell lui-même, cf. la belle biographie de Nigel Smith, Andrew Marvell, Londres et New Haven, Yale University Press, 2010.

13. HDNP, Archives Colijn, 0951/054/18, Gulbenkian à Colijn, 15 mars 1937, s.f.

14. BNM 10695, Representación a Carlos II de Francisco Manuel de Lira sobre la enemistad del Rey de Francia hacia España y otros asuntos de Estado, fo86.

15. KB, Knuttel, 11503 A, L'Europe esclave si l'Angleterre ne rompt pas avec ses fers (1677), p. 46.

16. Lille, $\mathrm{AD}$ du Nord, B 19595, Fonds Godefroy, Conférence de trois seigneurs wallons en forme de dialogues familiers sur les affaires du temps, Tournai, février 1678, s.f.

17. TNA, SP 9/200 (36), Archives Williamson, The Gold-Mine of France opened and stopped (1672), $f^{\circ} 2$. Il s'agissait là d'un thème récurrent de la propagande anti-française en Europe. Pour un exemple plus tardif dans le règne de Louis XIV, cf. Aix-en-Provence, Bibliothèque Méjanes, Le parallèle de Philippe II et de Louis XIV, Cologne, 1709. 
d'observation que constituait La Haye, Manuel de Lira s'accordait à voir en Louis $\mathrm{XIV}$ et ses ministres des " émules " de la " grandeur de la monarchie espagnole " ${ }^{18}$.

\section{Le rapprochement anglo-espagnol des années 1670}

À la suite du traité d'Aix-la-Chapelle (1668), où l'Espagne dut concéder des places fortes aussi importantes que Lille, Oudenarde, Douai et Tournai, la reine régente n'eut de cesse de tendre la main à l'ennemi britannique de jadis pour contrebalancer la puissance grandissante de la France. Dans les années 1620, c'est l'Angleterre qui avait cherché l'alliance de l'Espagne pour faire barrage à la France ${ }^{19}$. La situation était maintenant inversée : c'est l'Espagne, désormais, qui cherchait l'alliance de l'Angleterre, tout en menant une politique discrète de soutien aux catholiques persécutés en vertu des lois pénales du "Code Clarendon » voté à l'encontre des dissidents religieux entre 1662 et $1665^{20}$. La découverte par Kenneth Haley, il y a un demi-siècle, de la correspondance Pieter du Moulin dans les archives Fagel à La Haye, a donné lieu à de riches études sur le rapprochement anglo-néerlandais des années 1673-1678 ${ }^{21}$. En revanche, l'historiographie n'a rien produit d'équivalent sur le rapprochement anglo-espagnol des mêmes années. Plus généralement, les relations anglo-espagnoles restent un terrain à peu près vierge dans l'historiographie du XVII ${ }^{\mathrm{e}}$ siècle britanni$q^{2} e^{22}$. Il y a là un grand sujet qui appellera sans doute un jour une étude d'ampleur, à savoir ce que l'ambassadeur néerlandais à Londres Coenraad Van Beuningen appelait la «collusion" (collusie) entre l'Angleterre et l'Espagne. Plus exactement : entre le Parlement britannique et la Couronne d'Espagne ${ }^{23}$.

Un an à peine après la signature du traité séparé de Westminster (1674) entre l'Angleterre et la Hollande, le baron de Bergeyck (résident de l'Empereur Léopold à Londres) avait prévenu le gouverneur des Pays-Bas espagnols : «Il lui [Charles II]

18. BNM 10695, Representación a Carlos II de Francisco Manuel de Lira sobre la enemistad del Rey de Francia hacia España y otros asuntos de Estado, f $73 \mathrm{v}$.

19. Charles H. Carter, "Gondomar: Ambassador to James I", HJ, vol. VII, n 2, 1964 , pp. 189-208.

20. AGS, Estado 3972, Cuentas de embajadores y ministros en Inglaterra, s.f. L'ambassade d'Espagne à Londres déboursa 1126 livres sterling en ce sens en 1669 alors que le Parlement s'apprêtait à renouveler l'Acte sur les Conventicules de 1664.

21. Kenneth H. D. Haley, William of Orange and the English Opposition 1672-1674, Oxford, Clarendon Press, 1953. Steven Pincus, «From butterboxes to wooden shoes : the shift in English popular sentiment from anti-Dutch to anti-French in the 1670s ", HJ, vol. XXXVIII, n ${ }^{\circ} 2,1995$, pp. 333-361. Charles-Édouard Levillain, Vaincre Louis XIV. Angleterre-Hollande-France. Histoire d'une relation triangulaire, 1665-1688, Seyssel, Champ Vallon, 2010, pp. 204-206.

22. Pour de rares exceptions, cf. Albert J. Loomie, «Alonso de Cárdenas and the Long Parliament, 1640-1648 ", EHR, vol. LXXXXVII, n³83, 1982, pp. 289-307. Robert A. Stradling, "Spanish conspiracy in England, 1661-1663 ", EHR, vol. LXXXXVII, n 343, 1972, pp. 269-286. En français, voir le développement consacré par Stéphane Jettot à l'affaire Salinas (1677) in Représenter le Roi ou la Nation? Les parlementaires dans la diplomatie anglaise 1660-1702, Paris, PUPS, 2012, pp. 188-192.

23. NA, 3.01.18, Archives Fagel, inv. nr.251, Van Beuningen à Fagel, 9 décembre 1678, s.f. Sur Van Beuningen, la biographie définitive reste celle de Martinus Franken. M.A.M. Franken, Coenraad Van Beuningen's politieke en diplomatieke activiteiten in de jaren 1667-1684, Groningue, J.-B. Wolters, 1966. 
est impossible d'ignorer combien la conservation des Flandres lui importe ${ }^{24}$. " Les incursions croissantes des armées de Louis XIV dans les Pays-Bas espagnols au cours des années 1676-1678 avaient donné tout son sens à l'avertissement lancé par le représentant de l'Empereur en 1675. Les villes de Valenciennes (dans le Hainaut) et de Saint-Omer étaient tombées en 1677. Les convergences d'intérêts de Charles II et de Louis XIV étaient connues. Manuel de Lira continuait à penser que Charles II voulait se partager les dépouilles de l'Empire des Indes avec le Roi-Très-Chrétien ${ }^{25}$. Dans une Consulte de septembre 1677 sur les affaires d'Angleterre, le Conseil d'État espagnol se dit persuadé que le roi d'Angleterre cherchait à devenir " maître absolu de son Parlement ${ }^{26}$ ", à l'inverse, en somme, de son père Charles ${ }^{\text {er }}$, dont on disait volontiers dans les années 1640 qu'il vivait sous la "dépendance absolue " (absoluta dependencía) du Parlement ${ }^{27}$. À ce stade, le salut de l'Espagne dépendait de deux facteurs : d'une part l'alliance dynastique scellée entre Guillaume d'Orange et Marie Stuart (fille protestante du duc d'York), dont le marquis de Villa-Hermosa avouait beaucoup attendre pour renforcer la sécurité des Pays-Bas espagnols ${ }^{28}$; d'autre part la capacité du Parlement britannique à servir de relais aux exigences diplomatiques de la Couronne d'Espagne auprès des Stuarts.

\section{L’Angleterre face à la prise de Gand (1678)}

Il faut dire que, à partir de début 1678, les Espagnols remuèrent ciel et terre pour pousser le roi d'Angleterre à entrer en guerre contre Louis XIV et ainsi protéger les Flandres. Il fut même question, dans une Consulte de février 1678, de demander à la Couronne d'Angleterre de pouvoir utiliser certains de ses ports avant que le marquis de Bourgomaine, plus prudent, ne suggérât de se contenter des ports de Nieuport et d'Ostende, dans les Flandres occidentales ${ }^{29}$. Bourgomaine eut une conversation très franche avec le roi d'Angleterre, réclamant sans détours une assistance militaire contre la France. Charles II continuait à tergiverser, assurant l'ambassadeur de Carlos II de sa volonté de protection des Pays-Bas espagnols - ne serait-ce, disait-il, que pour "l'honneur» et le "bien » de la Couronne britannique - tout en refusant de se déclarer ouvertement contre Louis XIV. Le plus tranché, en un sens, était le duc d'York, aux yeux de qui la perte des Flandres risquait de faire perdre le trône d'Angleterre à son frère aîné - pas moins, assurait-il ${ }^{30}$. Vint ensuite la chute de Gand en mars 1678, une ville considérée comme un nœud stratégique par Charles-Quint lui-même ${ }^{31}$. L'émotion fut considérable en Angleterre où, selon Bourgomaine, «le

24. AGR T 100/2556/2, Secrétairie d'État et de guerre, Bergeyck à Fuenmayor, 12 mars 1675, s.f.

25. BNM 10695, Representación a Carlos II de Francisco Manuel de Lira sobre la enemistad del Rey de Francia hacia España y otros asuntos de Estado, f $76 \mathrm{v}$.

26. AGS, Estado 2134, Consulte sur les affaires d'Angleterre, 7 septembre 1677 NS, n 119, s.f.

27. BNM, MS 11262/38, Relación del estado presente de las cosas de Inglaterra, 1638-1652, f 5 .

28. Ibid., Villa-Hermosa au Prince d'Orange, (?) octobre 1677, n² 208, s.f.

29. AGS, Estado 2135, Consulte du Conseil d'État, 8 février 1678 NS, n 54 , s.f.

30. Ibid., Bourgomaine à Villa-Hermosa, 14 janvier $1678, n^{\circ} 58$, s.f.

31. Amiens, Bibliothèque municipale, MS 881, Papiers de Vault, Détails du siège de Gand (1678), f 2 . 
peuple accusa Charles II d'avoir laissé les choses dans cet état par faiblesse ${ }^{32}$ ». La Couronne d'Espagne ne pouvait que déplorer le sort malheureux des provinces du Nord, défendues par des soldats qui, à défaut d'être payés, se voyaient réduits à un état de "mendicitée ${ }^{33}$ ». Lointaine était l'époque glorieuse des tercios qui faisaient la loi dans le Nord de l'Europe, poussant jusqu'à Corbie en $1636^{34}$. Les sujets du Roi-Très-Catholique se disaient " abandonnés ${ }^{35}$ ".

Par rapport à l'Angleterre, la situation de la diplomatie espagnole fut compliquée par le déclenchement en septembre 1678 du complot papiste et du revirement qu'il entraina à la Chambre des Communes. En mars 1678, concomitamment à la prise de Gand, la Chambre basse avait demandé à Charles II de déclarer la guerre contre la France $^{36}$. Six mois plus tard, les mêmes parlementaires exigeaient du roi qu'il licenciât les troupes dont ils avaient pourtant réclamé la mobilisation en 1677. Pour l'Espagne, la nouvelle ne pouvait pas plus mal tomber. Confronté à de graves difficultés financières, le Roi-Très-Catholique ne pouvait compter que sur l'assistance financière de ses alliés. De l'aveu du comte d'Egmont, le rappel des quelque 4000 hommes engagés dans les Flandres risquait d'aggraver une situation jugée déjà " désastreuse ${ }^{37}$ ». On parlait parfois dans les milieux diplomatiques espagnols de la « faiblesse » du roi d'Angleterre ${ }^{38}$, notamment face à un Parlement dont on craignait qu'il ne " précipitât entièrement la destruction » des Stuarts ${ }^{39}$. Ou pire : qu'il ne « réduisît le gouvernement à une démocratie ${ }^{40} »$. C'est en ce sens que, dans une audience privée avec Charles II, les ministres espagnols lui firent entrevoir le grave danger que courait l'Angleterre en se retirant des Flandres ${ }^{41}$. Pris dans la tourmente du complot papiste, Charles II ne put évidemment donner suite aux requêtes de la Couronne d'Espagne.

\section{L'EXIL BRUXELLOIS DU DUC D’YORK (I 679)}

Venons-en maintenant à l'exil bruxellois du duc d'York et à son impact sur le rapprochement anglo-espagnol esquissé depuis 1673-1674. Cette période d'exil dura à peu près six mois, de mars à septembre 1679. Du point de vue international, deux dates méritent d'être rappelées : d'une part la signature du traité de Nimègue entre la France et l'Espagne en septembre 1678 ; d'autre part la mort, en septembre 1679, de Don Juan José, fils bâtard de Philippe IV d'Espagne. En l'état de faiblesse persistante

32. AGS, Estado 2135, Bourgomaine à Villa-Hermosa, 15 mars 1678, n 154 , s.f.

33. Ibid., Consulte du Conseil d'État, 3 avril 1678 NS, n 179 , s.f.

34. Pour ce sujet, nous renvoyons au grand classique de Geoffrey Parker, The Army of Flanders and the Spanish Road, 1567-1659, Londres, Cambridge University Press, 1975.

35. BNM 10695, Representación a Carlos II de Francisco Manuel de Lira sobre la enemistad del Rey de Francia hacia España y otros asuntos de Estado, fo86.

36. AGDHC, 15 mars 1678 , vol. V, p. 247.

37. AGR T 091/494, Ambassade d'Espagne à La Haye, Egmont à Manuel de Lira, 13 décembre 1678, ff.90-90v.

38. AGS, Estado 2135, Manuel de Lira au marquis de Balbases, 15 février 1678 NS, nº 69, s.f.

39. AGR T 091/494, Egmont à Lira, 28 novembre 1678, fo69.

40. Ibid., 5 décembre $1678, f^{\circ} 82$.

41. NA, 3.01.18, Archives Fagel, inv. nr.251, Van Beuningen à Fagel, 6 décembre 1678, s.f. 
de Carlos II, c'est Don Juan qui, de facto, tenait les rênes du gouvernement, allant jusqu'à exiler la reine régente Marianne d'Autriche à Tolède pour rester entièrement maître à bord. Ambassadeur d'Angleterre à Madrid, William Godolphin pensait que cette transition entraînerait de " grands bouleversements " à la Cour, où la concurrence pour la succession de Don Juan aiguisait de nombreux appétits ${ }^{42}$. C'est donc entre septembre 1678 et septembre 1679 que s'intercalèrent les six mois d'exil passés par le duc d'York à Bruxelles. Officiellement, la France et l'Espagne étaient en paix, mais nul ne croyait aux promesses de Louis XIV et, de fait, sa supposée «foi punique » faisait partie des traits de caractère les plus courants que lui prêtait la littérature anti-française ${ }^{43}$. Envoyé des États-Généraux à Madrid, Sébastien de Chèze observait un lourd silence à la Cour d'Espagne à propos du traité de Nimègue, vu comme une "paix honteuse" dont on cherchait sans grand espoir à laver l'affront ${ }^{44}$. Dans les faits, la guerre n'était pas tout à fait finie. Au moment où le duc d'York se trouvait à Bruxelles, le comte d'Avaux (ambassadeur de Louis XIV à La Haye) tentait de mettre la main sur la principauté de Liège. Il était même question d'une négociation secrète pour échanger les Pays-Bas espagnols contre la Catalogne, mais les ministres de Louis XIV n'y étaient pas favorables, jugeant ce troc inéquitable ${ }^{45}$. Les Pays-Bas espagnols demeuraient un avantage territorial supérieur.

\section{La crise de l'Exclusion, de Londres à Saint-Andrews : le fil de la chronologie}

Du point de vue britannique, la chronologie demande à être reconstituée avec d'autant plus de précision que l'accélération des événements, à partir de septembre 1678, fut compliquée par la révolte avortée des Covenantaires d'Écosse en juin 1679. C'est en ce sens qu'il y a bien une dimension "britannique ", et pas seulement anglaise, à la crise de l'Exclusion. Le duc d'York arriva dans les Pays-Bas fin mars 1679, quelques jours avant la démission du Lord Haut Trésorier Danby le 26 mars. La concomitance entre les deux événements ne devait évidemment rien au hasard. Envoyer son frère en exil pemettait à Charles II de mieux contrecarrer l'offensive que préparait le premier Parlement de l'Exclusion, réuni depuis le 6 mars 1679 après la dissolution, le 24 janvier, du " Parlement Cavalier ", ce "Long Parlement » qui avait siégé par intermittence depuis $1661^{46}$. Il existe à notre connaissance très peu de lettres entre Charles II et son frère au cours de cette période et, non sans raison, le duc d'York pouvait se plaindre de n'être traité « ni comme un frère ni comme un

42. BL, ADD MSS 47899, Godolphin à Bulstrode, 21 septembre 1679 NS, ff.250-250v.

43. Marchamont Nedham, Christianissimus Christianandus or Reasons for the Reduction of France to a more Christian State in Europe, Londres, 1678, p. 59.

44. NA 3.01.18, Archives Fagel, inv. nr.388, de Chèze à Fagel, 8 octobre 1678 NS, s.f. La France rendit à l'Espagne certaines des places fortes acquises lors du traité d'Aix-la-Chapelle (1668), comme Binche, Oudenarde et Courtrai, mais Louis XIV obtint des avantages territoriaux considérables, parmi lesquels la Franche-Comté ou, dans les Flandres et le Hainaut, Cassel, Ypres, Cambrai, Bouchain et Valenciennes. Il faut y ajouter La Trinité, dans les Antilles, où l'on produisait principalement du sucre.

45. TNA, SP 84/215, Meredith à Sunderland, 24 mai 1679 NS, f 126.

46. Annabel Patterson, The Long Parliament of Charles II, New Haven et Londres, Yale University Press, 2008. 
$\mathrm{ami}^{47}$ ». Ambassadeur d'Espagne à La Haye, Manuel de Lira le disait « abandonné de tous $^{48}$ ". Parmi les lettres de cette correspondance pour le moins maigre entre les deux frères se trouve dans les archives Godefroy à Lille ce qui ressemble à une copie d'un courrier, en date du 26 février 1679, de Charles II à son frère. De son écriture serrée, Denis Godefroy a laissé l'annotation suivante en haut de la lettre : « Lettre du roi d'Angleterre au duc d'York son frère portant ordre de se retirer et partir d'Angleterre. " Le simple fait que Godefroy ait pris la peine d'annoter la lettre pour en résumer le contenu témoigne de l'intérêt que présentait ce document aux yeux de l'archiviste lillois de Louis XIV. Voici donc le message de Charles II à son frère :

Je me suis déjà expliqué avec vous sur les raisons qui m’obligent à vous proposer de vous éloigner de moi en passant les mers comme je suis très fâché de l'occasion de votre absence vous pouvez aussi vous assurer qu'elle ne durera qu'autant qu'elle sera absolument nécessaire pour vos intérêts et mon service ${ }^{49}$.

«Autant qu'elle sera absolument nécessaire pour vos intérêts et mon service. " La formule ne peut être comprise qu'en étant replacée dans le fil de la chronologie. La crise de l'Exclusion avança par révélations successives. Le 27 avril 1679, un mois après l'arrivée du duc d'York à Bruxelles, la correspondance de son secrétaire catholique Edward Coleman fut ouverte. Une nouvelle rumeur commença à enfler : le frère du roi aurait engagé des négociations secrètes non seulement avec le roi de France mais avec le pape. N'y avait-il pas lieu de craindre, comme le suggérait un pamphlet publié en 1679, que « la cause catholique, comme les marrons dans la fable, ait toujours été dans le feu depuis le règne de la reine Marie ${ }^{50}$ ? ». En envoyant son frère sur le continent, Charles II avait, assez littéralement, tiré les marrons du feu. Car le premier bill d'exclusion fut rédigé le 15 mai 1679, quelques semaines après les révélations autour de la correspondance de Coleman. Le duc d'York exfiltré, la meilleure solution pour Charles II fut de suspendre le Parlement le 27 mai puis de le dissoudre le 12 juillet 1679. Ne l'oublions pas : le roi agissait en son Parlement, mais le Parlement ne pouvait exister sans son roi. Ou si, comme dans les années 1640, il existait sans son roi, le Parlement ne votait plus que des ordonnances, et non des lois ${ }^{51}$.

Un autre aspect essentiel du contexte politique du printemps-été 1679 fut la révolte avortée des Covenantaires d'Écosse. Cette révolte fut déclenchée par l'assassinat de l'archevêque de Saint-Andrews James Sharp par des presbytériens radicaux le

47. TBL, OSB-FB-190, Papiers Dartmouth, vol. II, duc d'York à Dartmouth, 25 juillet 1679, f 53 .

48. AGR T 091/493, Lira à Bourgomaine, 2 mai 1679 NS, f 145.

49. Lille, AD du Nord, Papiers Godefroy, B/19304, Charles II au duc d'York, 26 février 1679, folio unique.

50. CUL, Verney Tracts, Sel.2.16 (52), An Appeal from the Country to the City for the preservation of His Majesty's person, liberty, property and protestant religion, Londres, 1679, p. 2. Allusion à la fable de La Fontaine intitulée Le singe et le chat, publiée dans Les Fables choisies en 1679. La version originale de l'histoire se trouve dans les Fables d'Ésope, dont une nouvelle édition avait été publiée à Londres en 1678.

51. Pour des éclaircissements en français sur cette problématique, cf. Charles-Édouard Levillain, Un glaive pour un royaume. La querelle de la milice dans l'Angleterre du XVII siècle, Paris, Honoré Champion, 2013, chap. II passim, à paraitre. 
3 mai 1679. Une armée de 5000 hommes dirigée par le duc de Monmouth (fils bâtard de Charles II) fut aussitôt envoyée en Écosse. Écrivant depuis Londres aux négociateurs anglais présents à Nimègue, Orlando Bridgeman avoua prier Dieu que les Covenantaires ne reçoivent d'assistance ni de l'étranger ni des presbytériens d'Angleterre ${ }^{52}$. La révolte fut finalement écrasée dans le sang lors de la bataille de Bothwell Bridge le 22 juin 1679. Sans qu'il faille s'en étonner, l'épisode rappela à certains les prémisses de la guerre civile, à savoir la campagne militaire avortée menée par Charles ${ }^{\mathrm{er}}$ contre les presbytériens écossais en 1639-1640. Depuis Londres, le marquis de Bourgomaine y voyait une tentative de "destruction de la maison royale des Stuarts ${ }^{33}$ ». Comprenons bien : pour le Charles II finalement victorieux des Covenantaires, il ne s'agissait pas seulement de réussir là où son père avait échoué, mais de tenir en respect le duc de Monmouth, successeur potentiel du trône et héros des Whigs, qui risquait de faire un retour triomphal à Londres. Que Monmouth ait ressorti l'histoire de la « boîte noire » le lendemain même du départ du duc d'York pour les Pays-Bas ne devait évidemment rien au hasard ${ }^{54}$. Charles II était sur ses gardes. C'est également pour cette raison que le roi fut contraint de dissoudre le premier Parlement de l'Exclusion le 12 juillet 1679.

\section{L'Espagne face à la crise de l'Exclusion}

Revenons maintenant à la position de l'Espagne. Le déclenchement de la crise de l'Exclusion mit la Couronne d'Espagne devant un dilemme cornélien : du roi ou du Parlement, quel camp choisir? Un Parlement persécuteur de catholiques mais par ailleurs ennemi de la France ou un roi protecteur de catholiques mais par ailleurs ami de la France ? La question du statut du duc d'York, prince catholique exilé en terre espagnole, ne fit que cristalliser ces contradictions.

La première allusion à la possibilité d'un exil du duc d'York date d'une Consulte du Conseil d'État en date du 28 septembre 1678. Les conseillers du roi considéraient que trois possibilités s'offraient au duc d'York : la Hollande, la France ou les Pays-Bas espagnols. La Hollande leur apparaissait comme une solution peu crédible pour des raisons d'affinité religieuse. Les catholiques y étaient tolérés mais le Calvinisme restait malgré tout religion officielle et les relents d'anti-papisme étaient fréquents parmi la population. En 1666, par exemple, un pamphlet de facture orangiste avait parlé

52. TNA, SP 84/214, Bridgeman à Jenkins, 10 juin 1679, $\mathrm{f}^{\circ} 395$. Par une " assistance de l'étranger ", Bridgeman songeait principalement aux dissidents religeux anglais et écossais installés en Hollande.

53. AGR T 091/493, Bourgomaine à Lira, 17 juin 1679, s.f. Pour la révolte d'Écosse de 1639-1640, cf. Mark Fissel, The Bishops' Wars. Charles I's campaigns against Scotland, Cambride, Cambridge University Press, 1994 et le chef-d'œuvre d'érudition de Conrad Russell, The Fall of the British Monarchies, 1640-1642, Oxford, Clarendon Press, 1990. Pour le contexte écossais de la Restauration, Clare Jackson, Restoration Scotland 1660-1690. Royalist Politics, Religion and Ideas, Woodbridge, The Boydell Press, 2003.

54. AGS, Estado 3862, Bourgomaine à Carlos II, 29 mars 1679, s.f. Dans cette "boîte noire" (black box) sans doute fictive se serait trouvé le contrat de mariage de Charles II et de Lucy Walker, mère de James Scott duc de Monmouth. Monmouth avait toujours voulu se convaincre qu'il n'était pas le fruit d'une passade de Charles II, mais le gage d'un amour qui avait été scellé par un contrat en bonne et due forme. 
d'une opposition irréductible entre les « bons patriotes » et les " papistes » de France pourtant venus au secours de la Hollande (à l'instigation du Grand Pensionnaire Jean de Witt et de Coenraad Van Beuningen, alors ambassadeur à Paris) contre l'évêque de Münster ${ }^{55}$. En 1678, le peuple avait semble-t-il pris peur à l'annonce de l'arrivée de Marie de Modène duchesse d'York ${ }^{56}$. La deuxième solution était la France, où le duc d'York avait passé une partie de son exil dans les années 1650, partageant une vie plutôt désœuvrée entre le service des armées et les tentations de la vie parisienne. Bien que le duc d'York ait toujours paru " plus pro-français que le roi d'Angleterre ${ }^{57}$ ", un nouvel exil parisien paraissait difficile en raison des circonstances politiques. Il offrirait peut-être un avantage à Louis XIV, qui y verrait une occasion supplémentaire de «fomenter des divisions » en Angleterre, mais pas à Charles II, qui enverrait là un message contradictoire à son Parlement. Pour des raisons évidentes, l'Espagne n'y avait pas intérêt non plus. Ne pouvait-on craindre que, une fois en France, le duc d'York ne cherchât l'appui de Louis XIV pour "susciter une guerre civile ${ }^{58}$ " ?

Les Pays-Bas espagnols restaient donc, par déduction, la meilleure solution, au sens où chacun semblait y trouver son compte. Il restait cependant deux complications : la première venait du lien dynastique qui unissait les Oranges et les Stuarts depuis 1641. En 1677, le duc d'York avait été contraint d'offrir sa fille Marie en mariage à Guillaume III sous la pression conjointe de Charles II et de Danby : un mariage de raison, en quelque sorte, pour constituer un bloc anglo-néerlandais face à la France. En acceptant de recevoir le duc d'York à Bruxelles, l'Espagne courait malgré tout le risque de laisser les régents de la province de Hollande, par nature méfiants, soupçonner l'existence d'une liaison secrète entre les Oranges et les Habsbourgs d'Espagne. Ne disait-on pas de Van Beuningen, puissant régent d'Amsterdam et ambassadeur à Londres, qu'il ne souhaitait la paix que " pour désarmer le Prince et le réduire à un bourgeois d'Amsterdam ${ }^{59}$ ? "; Guillaume III, ce prince en république qui, avant une tentative avortée pour devenir duc de Gueldre (1675), s'était peut-être imaginé à la tête d'un "royaume de Batavie ${ }^{60}$ ". Ni roi de Batavie ni bourgeois d'Amsterdam, le Prince d'Orange demeurait dans une position intermédiaire et quelque peu indéfinissable qu'un rapprochement avec son oncle et beau-père d'Angleterre risquait de fragiliser. Au milieu des années 1670, alors que progressaient les négociations relatives à son mariage avec Marie, les prédicants avaient craint que le Prince d'Orange, tombant sous l'influence du duc d'York, ne mollisse dans sa lutte contre le papisme ${ }^{61}$.

55. KB, Knuttel 9391, Observatie van een Amsterdammer geschreven aan een goeden patriot op het subject van dezen tegenwoordige oorlog met Engeland (1666), p. 8.

56. AGS, Estado 3862, Consulte de la Jointe politique, 28 septembre 1678 NS, s.f.

57. Ibid.

58. AGR T 091/493, Bourgomaine à Lira, 11 avril 1679, fீ113.

59. AGS, Estado 2134, Bourgomaine à Villa-Hermosa, 18 juin 1677, $n^{\circ} 11$, s.f.

60. AGS Estado 8411, Manuel de Lira à Carlos II, 6 février 1675 NS, s.f. Pour plus de détails sur l'incident du duché de Gueldre, cf. Charles-Édouard Levillain, Vaincre Louis XIV, op. cit., pp. 250-254. En néerlandais, cf. M.W. Hartog, "Prins Willem III en de Hertogshoed van Gelderland 1673-1675 ", Bijdragen en Mededelingen der Vereniging Gelre, vol. LXIX, 1976-1977, pp. 125-155. En anglais, cf. Jonathan Israel, The Dutch Republic. Its Rise, Greatness and Fall, 1477-1806, Oxford, Clarendon Press, 1995, pp.816-818.

61. TNA, SP 84/205, Meredith à Williamson, s.d., f 178 . 
La deuxième complication peut se résumer à une question déjà évoquée à laquelle l'arrivée du duc d'York dans les Flandres donna une nouvelle substance : du roi ou du Parlement, qui choisir ? Devant les atermoiements de Charles II, l'intérêt de l'Espagne était de choisir le Parlement. Qui exactement, parmi les députés de l'opposition, reste difficile à dire. Il n'y avait pas à proprement parler de " parti espagnol ", ou même de "parti Habsbourg " à la Chambre des Communes, même si on croise par exemple le nom de Robert Thomas dans la correspondance du marquis de Bourgomaine, un proche de William Cavendish qui avait basculé dans l'opposition à partir de 1673. Tout au plus peut-on discerner une communauté d'intérêts autour d'un containment de la puissance de la France. D’après Sébastien de Chèze, il s'agissait pour les ennemis de Louis XIV " de s'adresser plutôt au Parlement et de les gendarmer contre le roi ${ }^{62}$ ».

\section{Bruxelles 1679 : réseaux et sociabilités}

Affinons maintenant l'analyse pour nous concentrer sur les six mois passés par le duc d'York à Bruxelles entre mars et septembre 1679. Et commençons par envisager la position de la Couronne d'Espagne par rapport au déroulement effectif de cet exil. Dès son arrivée à Bruxelles, le duc d'York écrivit au roi d'Espagne pour se placer sous sa protection, déclarant vouloir vivre incognito, à l'abri des regards ${ }^{63}$. Du côté espagnol, on ne peut exactement parler d'enthousiasme à l'idée de devoir accueillir le frère du roi. À La Haye, Manuel de Lira voyait d'un mauvais œil la présence d'un Stuart en terre espagnole alors que l'Espagne tentait précisément de resserrer ses liens avec l'Angleterre. Le Parlement risquait d'en prendre ombrage. Quant au duc d'York lui-même, Manuel de Lira se rendit compte en lui parlant qu'il n'était guère porté à un rapprochement avec la Couronne d'Espagne ${ }^{64}$. À Madrid, la prudence était de mise. On prenait acte de sa volonté de rester incognito, mais on demandait à Villa-Hermosa de chercher à en savoir plus sur les intentions réelles ou cachées du duc d'York. La main secrète de la France se cachait peut-être derrière ce transfert de Londres vers Bruxelles ${ }^{65}$. La seule exception à ce tableau plutôt sombre était Manuel Ponce de León, duc d'Arcos et d'Aveiro. Considéré par Godolphin comme l'un des princes de sang les plus importants de la monarchie espagnole, multi-propriétaire, bardé d'honneurs et proche du roi, dont il avait la confiance, le duc d'Arcos et d'Aveiro s'était déjà signalé par un remarquable don fait à Charles II d'Angleterre quelques années auparavant : trois splendides étalons élevés en Andalousie. L'anglophilie de ce grand d'Espagne était d'autant plus grande que son épouse descendait en ligne directe de la maison des Lancastres. Lorsqu'il apprit que le frère de Charles II en personne avait élu domicile à Bruxelles, le duc d'Arcos lui transmit aussitôt une lettre de bienvenue par l'intermédiaire de Godolphin ${ }^{66}$.

62. NA, 3.01.18, Archives Fagel, inv. nr.388, de Chèze à Fagel, 20 avril 1679 NS, s.f.

63. AGS, Estado 3862, duc d'York à Carlos II, 30 mars 1679 NS, s.f. Même message à l'intention du gouverneur des Pays-Bas espagnols Villa-Hermosa quelques jours avant, alors que le duc d'York se trouvait encore à La Haye. Ibid., 18 mars 1679 NS, s.f.

64. Ibid., Manuel de Lira à Carlos II, 28 mars 1679 NS, s.f.

65. Ibid., Consulte de la Jointe politique, 28 mars 1679 NS, s.f.

66. BL, ADD MSS 47899, Godolphin à Bulstrode, 14 mai 1679 NS, ff.225-227. 
Parmi les réactions espagnoles à l'installation du duc d'York à Bruxelles en mars 1679, la plus intéressante reste celle du marquis de Bourgomaine. Entre le 13 et le 29 mars 1679, l'ambassadeur d'Espagne à Londres rédigea trois longues dépêches à l'intention du roi et de ses ministres qui visaient en quelque sorte à fixer le cadre du séjour du duc d'York à Bruxelles. Essayons d'en dégager les principaux thèmes. Le premier est que Bruxelles restait un lieu d'exil malgré tout proche de Londres - une petite semaine de voyage à l'époque. À ce titre, les événements d'Angleterre continuaient à peser quotidiennement sur la situation du duc d'York. Malgré le relatif silence imposé par les circonstances, la séparation n'était pas totale entre les deux frères. Au moment où le duc d'York s'installa à Bruxelles en venant de La Haye, Bourgomaine observa que les affaires d'Angleterre étaient marquées par une "grande confusion ». "Envahi par la crainte ", Charles II semblait ne pouvoir "imprimer aucune marque en rien ", pendant que le duc de Monmouth, poussé par les Whigs, restait en embuscade ${ }^{67}$. La rivalité entre les deux ducs - d'un côté le "duc protestant " (comme l'appelaient les Whigs) et, de l'autre, le frère cadet héritier légitime du trône - restait l'un des moteurs de la crise de l'Exclusion. En ce sens, il s'agissait d'une crise dynastique autant que populaire.

Un deuxième thème important qui traverse la correspondance Bourgomaine est la question des fréquentations du duc d'York à Bruxelles. L'ambassadeur d'Espagne à Londres n'était pas naï : le duc d'York avait beau vouloir vivre dans la discrétion, ses moindres faits et gestes étaient surveillés par des espions mandatés par Shaftesbury et ses alliés. Si la Couronne d'Espagne ne donnait aucune consigne, laissant le duc d'York libre de ses occupations et de ses fréquentations, elle risquait de " perdre l'amour de cette assemblée [La Chambre des Communes] ${ }^{68}$ ". Les préconisations de Bourgomaine étaient donc très strictes : point de correspondance avec Vienne ou Rome en raison du risque d'une "nouvelle affaire Coleman "; point de contacts avec les catholiques anglais exilés en France ; point de contacts, surtout, avec les Jésuites, dont la supposée conspiration avait été au cœur des révélations truquées de Titus Oates en septembre $1678^{69}$; Titus Oates et son complice William Bedloe dont Godolphin rappela qu'ils avaient fréquenté le collège irlandais de Salamanque et dont les affabulations étaient décrites en Espagne comme une "fable forgée de toutes pièces" (a complete novela $)^{70}$. Pour les mêmes raisons, Bourgomaine déconseillait vivement à la Cour d'Espagne d'inviter le duc d'York à Madrid ${ }^{71}$. La meilleure solution restait l'isolement. Moins le duc d'York se mêlait de politique, mieux l'Espagne se portait.

Qu'en était-il maintenant du duc d'York lui-même ? Les archives existantes permettent de reconstituer une partie de ses activités et de ses fréquentations. De toute évidence, ce ne fut pas une période heureuse dans sa vie. Bruxelles n’avait ni le charme de la vie parisienne qu'il avait connue dans les années 1650 ni l'attrait d'une capitale politique comme Paris ou Vienne. Mis à l'index par son frère, plus ou moins boudé

67. AGS, Estado 3862, Bourgomaine à Carlos II, 29 mars 1679, s.f.

68. Ibid., 17 mars 1679 , s.f.

69. Titus Oates, A True Narrative of the Horrid Plot and Conspiracy of the Popish Party against the Life of his Sacred Majesty and Government and the Protestant Religion, Londres, 1679.

70. BL, ADD MSS 47899, Godolphin à Bulstrode, 29 juin 1679 NS, f $283 v$.

71. AGS, Estado 3862, Bourgomaine à Carlos II, 13 mars 1679, s.f. 
par les autorités espagnoles, peu contemplatif par nature, le duc d'York s'ennuyait sec. L'une des diversions peu heureuses qu'il s'accorda fut de rendre visite à sa fille Marie à La Haye, alors alitée après une fausse couche ${ }^{72}$. Il fut d'ailleurs accompagné par Charles-Henri de Lorraine-Vaudémont, fils de Charles IV de Lorraine et commandant des armées impériales. En raison de sa naissance et de son sang, le duc d'York faisait naturellement partie de ces réseaux de sociabilité transnationaux propres à la haute noblesse européenne, portée par une même conception de l'honneur et, souvent, une expérience partagée de la chose militaire ${ }^{73}$. C'est à ces compagnons d'exil ou de voyage que le duc d'York eut l'occasion de répéter tout le mal qu'il pensait d'un complot papiste "dénué de tout fondement ${ }^{74}$ ". Pour exister autant que pour tuer l'ennui, le duc d'York parvint à reconstituer, sinon une Cour, du moins un entourage de proches fidèles : majoritairement des catholiques, comme le duc de Norfolk $^{75}$, le comte de Peterborough ou encore Sir Henry Belasyze ${ }^{76}$. Mais l'appartenance confessionnelle ne constituait pas un absolu puisqu' on y trouvait également des huguenots comme Louis Duras comte de Feversham, un militaire de carrière qui, tel le comte de Souche dans l'Empire, faisait partie de cette petite diaspora de soldats protestants au service de princes catholiques ${ }^{77}$.

\section{L'exigence de la religion}

De Jacques II lui-même, il ne reste hélas que très peu de lettres de cette période, ce qui explique en grande partie le silence de ses biographes sur le sujet depuis le XVIII $^{\mathrm{e}}$ siècle. Publiée en 1828, la correspondance Hyde contient une lettre où le duc d'York explique à son neveu Lawrence Hyde qu'il ne changerait jamais d'avis en matière de religion : "Ce que j'ai fait n’a jamais été fait dans la précipitation. J'ai attendu de nombreuses années et me suis préparé à ce qui m’est arrivé et au pire qui peut encore m'arriver ${ }^{78}$." On reconnaît là chez le duc d'York une obstination

72. TNA, SP 84/215, Meredith à Sunderland, 13 avril 1679 NS, f 90 . De même 20 avril 1679 NS, f 98 .

73. Pour ce sujet, cf. Lucien Bély, La société des princes, Paris, Fayard, 1999. En anglais, de la plume d'un historien de la Lorraine, Jonathan Spangler, The Society of Princes. The Lorraine-Guise and the Conservation of Power and Wealth in seventeenth-century France, Farnham, Ashgate, 2009. Nous renvoyons également à la belle synthèse éditée en 1995 par Hamish Scott : The European Nobilities in the Seventeenth and Eighteenth centuries, Londres et New York, Longman, 1995.

74. NA, 3.01.18, Archives Fagel, inv. nr.228, Thomas Van Sarburgh à Fagel, 10 juillet 1679 NS, s.f.

75. AGS Estado 3862, Bourgomaine à Carlos II, 17 mars 1679, s.f.

76. Ibid., 13 mars 1679, s.f. TNA, SP 77/52, Bulstrode à Sunderland, 26 juin 1679 NS, fo $160 \mathrm{v}$.

77. AGS, Estado 3862, Bourgomaine à Carlos II, 13 mars 1679 NS, s.f. Aix-en-Provence, Bibliothèque Méjanes, MS 1042 (682), Mémoires de la Cour de l'Empereur en l'année 1670-1671, $\mathrm{f}^{\circ} 29$. Sur les soldats huguenots de l'Europe du second Xvir ${ }^{\mathrm{e}}$ siècle, cf. War, Religion and Service. Huguenot soldiering, 1685-1713, éd. Matthew Glozier et David Onnekink, Aldershot, Ashgate, 2007.

78. The Correspondence of Henry Hyde, Earl of Clarendon and his brother Lawrence Hyde, Earl of Rochester, Londres, 1828, vol. I, duc d'York à Lawrence Hyde, 24 juillet 1679 NS, p. 45. Le duc d'York avait épousé en première noce Anne Hyde, fille de Henry Hyde comte de Clarendon, qui devait servir en qualité de Lord Chancelier de Charles II entre 1660 et 1667. Pour plus de détails sur Lawrence Hyde, cf. Grant Tapsell, «Lawrence Hyde and the Politics of Religion in Later Stuart England », EHR, vol. CXXV, n 157, 2010, pp. 1415-1448. 
têtue qui, à partir de 1687, lui valut les ennuis que l'on sait. Le meilleur aperçu de la situation personnelle du duc d'York au cours de son exil bruxellois reste cependant celui que donne sa correspondance avec George Legge comte de Dartmouth. George Legge faisait partie des fidèles parmi les fidèles et devait d'ailleurs le rester jusqu'au bout. Entré au service du duc d'York en 1668, il avait fait partie des commandants de l'armée des Flandres, rejoignant les quelque 4000 hommes (dont 800 à Ostende) envoyés par Charles II après la chute de Gand en mars 1678. Élu de la ville portuaire de Portsmouth (dont il était le gouverneur) aux élections de 1679, Dartmouth s'était fait remarquer par ses positions en faveur du duc d'York dans un Parlement alors dominé par les Whigs, s'attirant de nombreuses inimitiés parmi ces derniers.

C'est par l'entremise de Dartmouth que le duc d'York restait régulièrement informé des évolutions des débats parlementaires. Une partie de cette correspondance a été publiée ${ }^{79}$; une autre ne l'est pas et reste aujourd'hui conservée dans le riche fonds James Osborn de la Beinecke Library. L'analyse se concentrera ici sur deux lettres écrites à deux mois d'intervalle, le 25 avril et le 25 juillet 1679. La première apporte un complément lumineux à la lettre déjà citée à Lawrence Hyde. Il est question de religion dans une perspective comparée Espagne-Hollande. Avant de s'installer à Bruxelles fin mars, le duc d'York avait fait une halte à La Haye avant de prendre la route du sud vers Breda. Il reconnut avoir été « bien traité » en Hollande. À Breda, disait-il, il n'y avait aucune raison que les catholiques viennent moins à lui qu'ailleurs. Car "dans ce pays, Dieu soit loué ", jugea-t-il, "on ne demande jamais à personne de quelle religion il/elle est ${ }^{80}$ ". Mais il restait malgré tout une grande différence entre un régime de tolérance favorable à une hétérogénéité confessionnelle et un régime uniconfessionnel tourné vers un catholicisme militant ${ }^{81}$. Lors de sa halte à La Haye, le duc d'York n'avait pas caché à Manuel de Lira qu'il souhaitait vivre en pays catholique en raison de la "dévotion " qu'il y trouverait et de la possibilité de célébrer la semaine sainte ${ }^{82}$. À Bruxelles, insista le duc d'York auprès de Dartmouth, les catholiques ne constituaient pas une minorité tolérée mais un groupe majoritaire. Or, avoua le duc d'York, " on ne peut pas être plus catholique que moi ${ }^{83}$ ".

Rédigée le 25 juillet 1679, la seconde lettre n'est pas sans rappeler, par son ton et son style, la lettre de la veille écrite à Lawrence Hyde. Le duc d'York se plaignait de sa condition, avouant "s'armer de patience " face au sentiment que la fin de son exil ne serait pas pour demain. Il faut reconnaittre que, au printemps 1679, les chances d'un retour rapide vers la mère-patrie semblaient diminuer de jour en jour. Le Parlement paraissait avoir fortement gagné en "puissance " depuis l'empêchement de Danby

79. The Manuscripts of the Earl of Dartmouth, Historical Manuscripts Commission, Eyre \& Spottiswoode, 1887-1896.

80. TBL, OBS-FB-190, Papiers Dartmouth, vol. II, duc d'York à Dartmouth, 25 avril 1679 NS, f 34 .

81. Pour plus de détails sur la question de la tolérance, cf. Willem Frijhoff, "Religious toleration in the United Provinces: from case to model ", in Calvinism and Religious Toleration in the Dutch Republic, éd. R. Po-Chia Hsia et Henk van Nierop, Cambridge, Cambridge University Press, 2002, chap.3, passim. Les travaux de Willem Frijhoff ont permis de nuancer l'idée longtemps répandue des catholiques néerlandais comme une minorité inexistante dans une République à dominante calviniste.

82. AGS, Estado 3862, Manuel de Lira à Carlos II, 28 mars 1679 NS, s.f.

83. TBL, OBS-FB-190, Papiers Dartmouth, vol. II, duc d'York à Dartmouth, 25 avril 1679 NS, f’34. 
et, d'après Bourgomaine, les Whigs nourrissaient désormais le dessein "d'abattre l'autorité du roi $^{84}$ ». Après la dissolution du Parlement le 12 juillet 1679, le duc d'York pouvait se convaincre que " toutes choses tendaient vers une république ${ }^{85}$ ". La crainte d'un éternel retour de l'Interrègne : telle était l'une des obsessions les plus tenaces du duc d'York. Il n'hésitait pas, au demeurant, comme son père avant lui sur l'échafaud, à assumer la posture du martyre prêt à mourir pour sa religion : "Si l'occasion se présentait, j'espère que Dieu me donnerait la grâce de la mort, comme celle du bannissement, pour la vraie religion catholique. Ce que j'ai fait, je l'ai fait sans précipitation, après mûre réflexion ${ }^{86}$."

\section{CONCLUSION}

Cette phrase du duc d'York où perce un mélange de désespoir et d'entêtement nous mène au terme de l'analyse. À travers cet épisode bruxellois oublié de la vie du futur Jacques II se dessinent en filigrane certains traits de personnalité, certaines postures qui devaient ensuite dominer son règne : une fidélité absolue au catholicisme, la mémoire blessée du régicide, la hantise d'une atteinte à l'autorité de la monarchie et - c'est sans doute là le plus intéressant lorsque l'on pense à ce qui devait arriver dans la plaine de Salisbury en novembre 1688 - une certaine résignation face à l'adversité. Étrange mystère, chez le duc d'York, que ce mélange d'obstination, parfois même de raideur, et de renoncement à l'action au moment où survient l'inattendu. Peut-être que, contrairement à son frère, le duc d'York n'était tout simplement pas doué pour la politique : "regnandi nescius ", comme devait l'écrire de façon quelque peu lapidaire un correspondant du Grand Pensionnaire Fagel en $1688^{87}$.

Au-delà de la personne du duc d'York se pose la question plus large des relations anglo-espagnoles au cours de la crise de l'Exclusion. Remplaçant le marquis de Bourgomaine à Londres en juillet 1679, Don Pedro Ronquillo poursuivit une même politique de lobbying auprès des Whigs. N'hésitant pas à comparer le complot papiste à un " abîme " (abismo) qui avait fait perdre à " la famille royale l'amour, le respect et la vénération du peuple " et déplorant cette "tragédie inhumaine " qui frappait les catholiques d'Angleterre ${ }^{88}$, Don Pedro Ronquillo n'en restait pas moins un ferme allié des Whigs, dont il se servait comme un levier pour tenter de pousser Charles II à une intervention militaire dans les Flandres. Aux yeux de Don Pedro Ronquillo, les Flandres constituaient bien " la clé de la monarchie universelle ${ }^{89}$ ": une formule reprise dans un tract diffusé en 1681, dont la publication devait à

84. AGS, Estado 3865, Bourgomaine au roi, 7 avril 1679, s.f.

85. TBL, OBS-FB-190, Papiers Dartmouth, vol. II, duc d'York à Dartmouth, 29 novembre 1679 NS, f 58. Le duc d'York se trouvait alors à Édimbourg.

86. Ibid., 25 juillet $1679 \mathrm{NS}, \mathrm{f}^{\circ} 52$.

87. NA, 1.10.29, Archives famille Fagel, inv.nr. 2019 (1688), nº 68, anon. à Fagel, 8 octobre 1688, s.f.

88. AGR, I 115, Manuscrits divers, MS 950-951, Relación y Memorias históricas y politicas, ff. $279 \mathrm{v}-280$.

89. AGS, Estado 3866, Don Pedro Ronquillo à Carlos II, 2 juin 1681, s.f. 
l'imprimeur baptiste Francis Smith, un protégé de Shaftesbury qui faisait partie des piliers de la radicalité $w h i g^{90}$. Ce n'était pas le moindre paradoxe de voir le représentant à Londres du Roi-Très-Catholique travailler main dans la main avec un baptiste venu du Nord de l'Angleterre pour tenter de faire sortir Charles II de sa prudente neutralité. Un ambassadeur d'Espagne venant expliquer à la nation anglaise ce qu'il faut entendre par "monarchie universelle » : la boucle était pour ainsi dire bouclée.

\author{
Charles-Édouard LEVILLAIN \\ Université de PARIS VII DENIS DIDEROT
}

90. The Last Memorial of the Spanish Ambassador faithfully translated into English, Londres, 1681, page unique. J.S.T. Hetet, A literary underground in Restoration England: printers and dissenters in the context of constraints, 1660-1689, Thèse inédite de l'Université de Cambridge, 1987, p. 79. 